\title{
Giant Cyclones in Gaseous Discs of Spiral Galaxies
}

\author{
A. M. Fridman, O. V. Khoruzhii, E. V. Polyachenko ${ }^{a}$ \\ A. V.Zasov, O. K. Sil'chenko ${ }^{\text {b }}$ \\ V. L. Afanas'ev, S. N. Dodonov, A. V. Moiseev ${ }^{c}$ \\ ${ }^{a}$ Institute of Astronomy, 48 Pyatnitskaya Str., Moscow, 109017 Russia \\ ${ }^{\mathrm{b}}$ Sternberg Astronomical Institute, Universitetskii pr. 13, Moscow, 119899 Russia \\ ${ }^{\mathrm{c}}$ Special Astrophysical Observatory, Nizhnyi Arkhyz, Karachaevo-Cherkessia, \\ $35714^{7}$ Russia
}

\begin{abstract}
We report detection of giant cyclonic vortices in the gaseous disc of the spiral galaxy NGC 3631 in the reference frame rotating with the spiral pattern. A presence of such structures was predicted by the authors for galaxies, where the radial gradient of the perturbed velocity exceeds that of the rotational velocity. This situation really takes place in NGC 3631 .
\end{abstract}

\section{Introduction}

In previous papers [1], [2] authors have shown that besides the spiral arms, well known for more than one and a half century in spiral galaxies as structures in the brightness distribution, there also exist structures, revealing themselves in the velocity field. The latter structures have the appearance of giant anticyclonic vortices: their rotation is opposite to the rotation of the galactic disc. The centers of these vortices are located near the zero points of the force field (Lagrange points $L_{4}$ and $L_{5}$, see [3]). In spiral galaxies these points are fixed in the close vicinity of the corotation circle, where the velocity of the rigid-body rotating spiral pattern coincides with the velocity of the differentially rotating disc. Being in such a position, the centers of the anticyclones turn out to be fixed with respect to the spiral arms and stationary if the spiral structure is stationary. If the spiral structure develops due to some instability in the disc, then the arms and vortices arise simultaneously and grow with the same growth rate [4]. As a result a unified spiral-vortex structure forms. 
The growth of the perturbation amplitude leads rather often to the saturation of the instability, after which a spiral-vortex structure becomes stationary. From the very beginning this scenario implies that spiral arms in discs of galaxies have a wave nature, that satisfies modern theoretical conceptions [5] and agrees well with the results of the velocity field analysis in spiral galaxies $([2],[6])$.

A typical rotation velocity field of a galactic disc is represented schematically in Fig. 1a. There is no way to close the trajectories of particles in the laboratory (inertial) reference frame by any radial component of the velocity (arrows radially directed in Fig. 1a). The situation is opposite in the rotating reference frame, where anticyclonic motion takes place under condition of appropriate azimuthal variation of the radial component (see Fig. 1b). Note that in the latter case it is not necessary to have large radial velocities to form a vortex near the circle corotating with the reference frame due to small values of the azimuthal velocities in this region.

It is not accidental that the radial velocity was chosen as a periodic function of the azimuth: in the spiral density wave such periodicity should take place both for the radial $\tilde{V}_{r}$ and for the azimuthal $\tilde{V}_{\phi}$ perturbed velocities. Arrows

for $\tilde{V}_{\phi}$ are not shown in Fig.1. Taking into account the perturbed azimuthal velocity will not change the picture qualitatively, if

$$
\left|\partial \tilde{V}_{\phi} / \partial r\right|<\left|d V_{\text {circ }} / d r\right|
$$

where $V_{\text {circ }} \equiv V_{\text {rot }}-\Omega_{r f} r$ is the circular velocity in the reference frame rotating with the angular velocity $\Omega_{r f}$. Note that even a weak inequality is quite enough to form only anticyclonic vortices like in Fig. 1b (for more details see [7]). Under the opposite condition:

$$
\left|\partial \tilde{V}_{\phi} / \partial r\right|>\left|d V_{\text {circ }} / d r\right|
$$

not only anticyclonic, but also cyclonic vortices can appear [8]. In the next section we will consider both these cases in a schematic model of a galactic disc with a well-defined two-armed spiral structure (Grand-Design galaxy).

\section{Qualitative description of anticyclone and cyclone formation in a disc of Grand-Design galaxy}

Let us assume that two-armed Grand-Design spiral structure exists in a differentially rotating gaseous disc. Then the functions describing the perturbed 
surface density and velocity components, related to the density wave, may be approximated in the form:

$$
\begin{gathered}
\tilde{\sigma}(r, \varphi)=C_{\sigma}(r) \cos \left[2 \varphi-F_{\sigma}(r)\right], \\
\tilde{V}_{r}(r, \varphi)=C_{r}(r) \cos \left[2 \varphi-F_{r}(r)\right], \\
\tilde{V}_{\varphi}(r, \varphi)=C_{\varphi}(r) \cos \left[2 \varphi-F_{\varphi}(r)\right] .
\end{gathered}
$$

As we consider the momentary picture of the spiral structure, a time dependence is absent in the equations above.

It is natural to ask, whether it is possible to use expressions (3)-(5) for the description of the dynamics of spiral density waves with a finite amplitude. We have checked it in [6], [14] by the example of the galaxy NGC 3631, where cyclones have been found just now (see Section III below). The operating sequence of the check was the following. First, we analysed the azimuthal expansion in the harmonic series of the observed surface brightness of this galaxy at different galactocentric radii. A domination of the second Fourier harmonic over others (see Fig. 1 in [14]) proved the correctness of the approximation expressed in Eq.(3).

Then the observed line-of-sight velocity field $V^{o b s}(r, \varphi)$ was analysed. This velocity can be expressed as (see [2], [9]):

$$
V^{o b s}(r, \varphi)=V_{s}+V_{\varphi}(r, \varphi) \cos \varphi+V_{r}(r, \varphi) \sin \varphi \sin i+V_{z}(r, \varphi) \cos i .(6)
$$

Here $i$ is an inclination angle (angle between the rotation axis of galaxy and line of sight) and $V_{s}$ is systemic velocity of a galaxy (velocity of the center of mass of the galaxy).

Within the frame of the present model

$$
\begin{gathered}
V_{r}(r, \varphi)=\tilde{V}_{r}(r, \varphi) \\
V_{\varphi}(r, \varphi)=V_{\text {rot }}(r)+\tilde{V}_{\varphi}(r, \varphi) \\
V_{z}(r, \varphi)=\tilde{V}_{z}(r, \varphi)=C_{z}(r) \cos \left[2 \varphi-F_{z}(r)\right],
\end{gathered}
$$

where $\tilde{V}_{r}, \tilde{V}_{\varphi}$ are determined by (4) and (5). Substituting (7) in (6) one obtains the model representation of the Fourier expansion for $V^{o b s}(r, \varphi)$.

Equating the coefficients of the model expansion to the coefficients calculated from observational data,

$$
V^{o b s}(r, \varphi)=V_{s}+\sum_{n}\left(a_{n}^{o b s} \cos n \varphi+b_{n}^{o b s} \sin n \varphi\right)
$$


we obtain the following set of relations between unknown characteristics of the vector velocity field of the galaxy and the Fourier coefficients of the observed line-of-sight velocity field:

$$
\begin{gathered}
V_{\text {rot }}+\frac{1}{2}\left(C_{r} \sin F_{r}+C_{\varphi} \cos F_{\varphi}\right)=a_{1}^{o b s}, \\
-C_{r} \cos F_{r}+C_{\varphi} \sin F_{\varphi}=2 b_{1}^{o b s} \\
C_{z} \cos F_{z} \cos i=a_{2}^{o b s} \\
C_{z} \sin F_{z} \cos i=b_{2}^{o b s} \\
-C_{r} \sin F_{r}+C_{\varphi} \cos F_{\varphi}=2 a_{3}^{o b s} \\
C_{r} \cos F_{r}+C_{\varphi} \sin F_{\varphi}=2 b_{3}^{o b s}
\end{gathered}
$$

From (9)-(14) we see that the perturbed velocity components $\tilde{V}_{r}, \tilde{V}_{\varphi}$ and $\tilde{V}_{z}$, written in the form of (4), (5) and (7) give contributions only in the first three harmonics of the Fourier expansion. This is really observed in the case of NGC 3631 (see Fig. 3 in [6]), where the first three Fourier harmonics of the lineof-sight velocity field dominate over the higher-order harmonics. This gives evidence for the correctness of the representation of the perturbed velocity components in the form (4), (5) and (7) for the galaxy NGC 3631.

To draw schematically the spiral-vortex structure in different reference frames we need relations between the phases $F_{r}(r), F_{\varphi}(r)$ and $F_{\sigma}(r)$. These relations were derived in [9] from the set of linearized equations. In spite of this simplification, an excellent qualitative agreement was found between the phase of the "modified" third harmonic (see below) of the observed line-of-sight velocity field and $F_{\sigma}(r)$ (see Fig. 5 in [6]), which enables to use the phase relations obtained in [9] for a qualitative description of the residual velocities. The modified third harmonic is a two-armed spiral with phase $F_{3}-\pi / 2$, i.e. $\propto \cos \left(2 \varphi-F_{3}+\pi / 2\right)$, where $F_{3}$ is the phase of the original radial-velocity-field third harmonic.

Below a list of these relations is presented.

a) Relations between the phases of the radial velocity $\tilde{V}_{r}$ and the surface density perturbation $\tilde{\sigma}$.

- On the corotation circle $r=r_{c}$ :

$$
F_{r}=F_{\sigma}-\frac{\pi}{2}
$$


whence

$$
\tilde{V}_{r}\left(r_{c}, \varphi\right)=-C_{r}\left(r_{c}\right) \sin \left[2 \varphi-F_{\sigma}\left(r_{c}\right)\right]
$$

i.e. on the corotation circle the extrema of $\tilde{V}_{r}$ coincide with the zeroes of $\tilde{\sigma}$ and vice versa.

- Inside the corotation circle, $r<r_{c}$,

$$
F_{r}=F_{\sigma}+\pi
$$

whence

$$
\tilde{V}_{r}(r, \varphi)=-C_{r}(r) \cos \left[2 \varphi-F_{\sigma}(r)\right], \quad r<r_{c}
$$

i.e. inside the corotation circle the maxima of $\tilde{V}_{r}$ coincide with the minima of $\tilde{\sigma}$ and vice versa.

- Outside the corotation circle, $r>r_{c}$,

$$
F_{r}=F_{\sigma}
$$

whence

$$
\tilde{V}_{r}(r, \varphi)=C_{r}(r) \cos \left[2 \varphi-F_{\sigma}(r)\right], \quad r>r_{c}
$$

i.e. outside the corotation circle the maxima and minima of $\tilde{V}_{r}$ coincide, respectively, with the maxima and minima of $\tilde{\sigma}$.

b) Relation between the phases of the azimuthal velocity $\tilde{V}_{\varphi}$ and the surface density $\tilde{\sigma}$.

- In all cases, both on the corotation circle, $r=r_{c}$, and out of it, $r \neq r_{c}$, we have

$$
F_{\varphi}=F_{\sigma}+\frac{\pi}{2}
$$

therefore

$$
\tilde{V}_{\varphi}(r, \varphi)=C_{\varphi}(r) \sin \left[2 \varphi-F_{\sigma}(r)\right]
$$

i.e. the extrema of $\tilde{V}_{\varphi}$ coincide with the zeroes of $\tilde{\sigma}$ and vice versa throughout the disc.

Using the expressions (16), (18), (20), and (22) we can draw a scheme for the velocity field with vortices overlayed on the scheme of the surface density distribution (Fig. 2). In Fig. 2b we can see the presence of cyclones and anticyclones in the field of residual velocity (that corresponds a reference frame locally corotating with the disc at each radius, $V_{\text {circ }}(r)=0$ ). This fact by 
itself does not depend on the amplitude of the spiral density wave, although the sizes of the vortices depend on this amplitude.

Lindblad and Langebartel [10] were the first to calculate the field of displacements of the stars in the gravitational potential of a bar. The form of the star displacement field resembles the system of two cyclones and two anticyclones, which is similar our schematic Fig. 2b. Refering on [11] Lynden- Bell [12] has noted the existence of cyclonic and anticyclonic trajectories in Fig. 9 of [10]. A similar picture of four vortices in the velocity field of a gaseous disc - two cyclones on the bar and two anticyclones between the spiral arms - was obtained in a recent paper [13].

In the reference frame rotating with the angular velocity $\Omega_{p h}$ of the two-armed spiral pattern the picture of vortices differs qualitatively from the previous one. In the case (1) when the gradient of the perturbed azimuthal velocity is less than the circular velocity gradient, i.e. the anticyclonic shear dominates over the cyclonic one, the appearance of cyclones is impossible - we can see only two anticyclones with centers near the corotation circle between two spiral arms (Fig. 2c). In the opposite case (2) when the gradient of the perturbed azimuthal velocity dominates over the circular velocity gradient, the location of the cyclones is determined by the place of this domination. Cyclones can survive almost in the same places as in Fig. 2b, i.e. on the corotation circle, but their sizes will be smaller. There are also two other variants when the cyclone center moves either inside or outside the corotation circle along the zero lines of the radial velocity. Finally, both last these variants can be realized simultaneously. In this case four cyclones exist in the velocity field as is shown in Fig. 2d.

\section{Discovery of Giant Cyclones in the Gaseous Disc of the Spiral Galaxy NGC 3631 with the $6 \mathrm{~m}$ telescope in SAO}

Finding giant vortices in a spiral galaxy requires the reconstruction of the vector velocity field in the reference frame rotating with the angular velocity of the spiral pattern. Thus, first of all the corotation radius should be determined. In this paper for NGC 3631 we use the position of the corotation derived in [14] on the base of the Fourier analysis of the observed line-of-sight velocity field. The observations of NGC 3631 were carried out with the scanning FabryPerot interferometer with Russian $6 \mathrm{~m}$ telescope. The details of observations were described in [6].

It is necessary to determine $\tilde{V}_{r}$ and $V_{\varphi}=V_{r o t}+\tilde{V}_{\varphi}$, i.e. to find five unknown functions: $V_{\text {rot }}(r), C_{r}(r), C_{\varphi}(r), F_{r}(r), F_{\varphi}(r)$ (see Eqs. (4), (5), and (7)). These five functions are connected with the characteristics of the observed velocity 
field by the four relations (9), (10), (13), (14). An additional condition required to close the system should have a theoretical origin. Unfortunately, up to now a reliable condition valid for any density wave amplitude is not available. Several possibilities discussed in the literature [2], [9] have limited applicability. To overcome this difficulty, we propose the following approach.

Among the functions listed above, $V_{\text {rot }}(r)$ could be the most reliably estimated on the base of independent observational data. For this purpose the equilibrium condition of the gaseous disc rotating in a gravitational potential $\Psi$ can be used

$$
\frac{V_{r o t}^{2}}{r}=\frac{\partial \Psi}{\partial r}
$$

The latter is determined from the mass distribution in a galaxy or its surface brightness maps assuming a costant mass-to-light ratio to be known. To calculate the right hand side of the equation (23) we use a three-component dynamical model of a spiral galaxy similar to [15]. In spite of the roughness of the model, the $V_{\text {rot }}$ curve found by this method is in the region between $\left(a_{1}\right)_{\min }$ and $\left(a_{1}\right)_{\max }$ (see Fig. 3 ; a peculiarity of the thick curve is described below).

The same result is obtained by another way. From equation (9) it follows that the difference $\left|a_{1}-V_{\text {rot }}\right|$ cannot exceed the amplitudes $C_{r}$ and $C_{\varphi}$, which, in turn, are connected by equations (10), (13) and (14) with the Fourier coefficients $b_{1}^{o b s}, a_{3}^{o b s}$, and $b_{3}^{o b s}$, determined from our observations. In Fig. 4 the radial behaviour of $b_{3}^{o b s}-b_{1}^{o b s}=C_{r} \cos F_{r}$ and $b_{3}^{o b s}+b_{1}^{o b s}=C_{\varphi} \sin F_{\varphi}$ is demonstrated. The extrema of these functions allow to estimate the amplitudes $C_{r}$ and $C_{\varphi}$. According to Fig. 4, one can conclude that in NGC 3631 a maximum value of the amplitude of the residual velocities can reach at $60 \mathrm{~km} / \mathrm{s}$, i.e.

$$
\left|a_{1}-V_{\text {rot }}\right|_{\max } \leq 60 \mathrm{~km} / \mathrm{s}
$$

The conditions (23) and (24) do not allow to calculate the function $V_{\text {rot }}(r)$ exactly. Nevertheless, they set limits on the variations of both the amplitude and the form of $V_{\text {rot }}(r)$. Within these limits, we choose a set of trial curves (Fig. 3) and analyse the velocity field obtained from Eqs. (9)-(14) for a given $V_{\text {rot }}(r)$.

The general views of the restored velocity fields are qualitatively similar for the full set of the rotation curves. In particular, in all cases, except the most extreme ones, distinct areas of anticyclonic as well as cyclonic shear flows exist. However, only rare examples demonstrate the presence of areas with trapped fluid particles moving along stream lines bounded by closed separatrices. Such behavior could be a natural consequence of the quasi-stationary character of 
the density wave. It is an argument in favour of choosing just these examples of $V_{\text {rot }}(r)$ as the best-corresponding to the real dynamical model of the galaxy. The best example of $U_{\text {rot }}$ from the point of view of the stationarity of the stream lines is shown by the thick curve in Fig.3. In other examples the vortices have not close separatrices and their stream lines are spirals - strongly inwards or outwards.

In Fig. 5 we can see the amplitudes of radial $\left(\tilde{V}_{r}\right)$ and azimuthal $\left(\tilde{V}_{\varphi}\right)$ components of the residual velocity. These amplitudes, $C_{r}$ and $C_{\varphi}$ were obtained from four equations (9), (10), (13), and (14) after substituting in them $V_{\text {rot }}(r)$ in the form drawn in Fig. 3 by the thickest line.

Using the functions $C_{r}$ and $C_{\varphi}$ in Fig.5 let us show, that the necessary condition (2) of the cyclone formation is satisfied in the galaxy NGC 3631 outside and inside the corotation circle and is not satisfied on the corotation circle. In the reference frame, rotating with the angular velocity of the spiral pattern $\left(\Omega=\Omega_{p h}\right)$, the inequality (2) can be rewritten in the form

$$
\left|\frac{\partial \tilde{V}_{\varphi}}{\partial r}\right|>\left|\frac{d V_{\text {circ }}}{d r}\right| \equiv\left|\frac{d}{d r}\left(V_{\text {rot }}-\Omega_{p h} r\right)\right| .
$$

Substituting here the expression (5) for $\tilde{V}_{\varphi}$, we obtain:

$$
\left|C_{\varphi}^{\prime} \cos \left(2 \varphi-F_{\varphi}\right)+F_{\varphi}^{\prime} C_{\varphi} \sin \left(2 \varphi-F_{\varphi}\right)\right|>\left|V_{c i r c}^{\prime}\right|
$$

where "prime" denotes the derivative with respect to $r$.

In order that cyclones should be possible the condition (26) should be fulfilled at least near the center of the vortex. In the centers

$$
V_{r}=\tilde{V}_{r}=C_{r} \cos \left(2 \varphi-F_{r}\right)=0
$$

This equation gives a possibility to reduce the left hand side of inequality (26) to a form more suitable for an estimation.

In the vicinity of the center of the cyclone in residual velocities (Fig. 2 b) on the corotation circle from (15) and (21) we have $F_{\varphi}=F_{r}+\pi$. Thus with the help of (27), the condition (26) is reduced to

$$
\left|F_{\varphi}^{\prime} C_{\varphi}\right|>\left|V_{c i r c}^{\prime}\right|
$$

As follows from Fig. $5 \mathrm{~b}$ at the corotation radius $(r \approx 42 \operatorname{arcsec}) C_{\varphi} \approx 0$. Hence, as the value of $F_{\varphi}^{\prime}$ is very small the inequality (28) cannot be fulfilled. 
In the case of "external" cyclones (see Fig. 2d) from (19) and (21) we have $F_{\varphi}=F_{r}+\pi / 2$. Then using (27) from (26) we obtain

$$
\left|C_{\varphi}^{\prime}\right|>\left|V_{\text {circ }}^{\prime}\right|
$$

This relation explains the coincidence of the location of the external cyclones in NGC $3631(52<r<59$ arcsec in Fig. 7) with the region of rapid growth of $C_{\varphi}(50<r<60$ arcsec in Fig. $5 \mathrm{~b})$.

In the case of the "internal" cyclones from (17) and (21) it follows $F_{\varphi}=$ $F_{r}-\pi / 2$. Then in this case the condition (26) also has the form (29). According to Fig. 5b it is fulfilled in the region $22<r<31$ arcsec, which really contains the internal cyclone region $25<r<30 \operatorname{arcsec}$ (Fig. 7).

The vector velocity field of the galaxy NGC 3631, when $V_{\text {rot }}(r)$ is taken in the form presented in Fig. 3 by the thickest line, is shown in Figs. 6 and 7. In the figures some streamlines of the velocity fields are presented. If the velocity field is stationary the trajectories of fluid particles coincide with the correspondent streamlines. One can see that the residual velocity field of NGC 3631 (Fig. 6) corresponds to that of Fig. 2b and the full velocity field in the reference frame of the spirals (Fig. 7) corresponds to either of two cases presented in Fig. 2d when the centers of two cyclones lie outside the corotation circle. Internal cyclones in the case shown in Fig. 7 are not enveloped by close streamlines.

\section{Conclusions}

I. An analysis of velocity fields in Grand-Design galaxies shows that:

1) the field of residual velocities contains two cyclones and two anticyclones with centers on the corotation circle;

2) in the reference frame rotating with the spiral pattern the velocity field belongs to one of two types:

a) under the condition (1) it contains only two anticyclones with centers near the corotation circle;

b) under the condition (2) besides the anticyclones the field contains also either two or four cyclones. In the former case the cyclone centers lay either

on, or outside, or inside the corotation circle. In the latter case, two pairs of cyclones appear with centers inside and outside the corotation circle.

II. Our analysis of the velocity field data obtained by our team at the $6 \mathrm{~m}$ 
telescope in the Special Astrophysical Observatory of the Russian Academy of Sciences shows that the Grand-Design galaxy NGC 3631 belongs to the type b).

\section{Acknowledgement}

We thank V. I. Arnold, B. V. Chirikov, V. L. Polyachenko, M. I. Rabinovich, Ya. G. Sinai, and specially G. Contopoulos for fruitful discussions. We thank also J.Bolesteix for his kind placing at our disposal a collection of interferometric filters.

This work was performed under partial financial support of RFBR grant 99-02-18432, grant "Leading Scientific Schools" 96-15-96648, and the grant "Fundamental Space Researches. Astronomy" for the 1999 1.2.3.1 and 1.7.4.3. 


\section{Figure Captions}

Figure 1. A scheme for the anticyclonic vortices formation in a reference frame rotating with an arbitrary angular velocity. Unperturbed rotation velocities and radial perturbed velocities are marked by solid arrows and dashed arrows correspondingly. a) In the laboratory system of coordinates the rotation velocity of the disc varies with radius without change of sign. In this case the radial perturbed velocity can not provide a vortex formation despite the regular change of its sign along azimuth. b) In a rotating reference frame, where the dotted circle is at rest, the same field of radial perturbed velocities as in Fig.1a participates in the anticyclone formation.

Figure 2. A scheme for the velocity field with vortices in different reference frames. Vectors of unperturbed rotation velocity field are shown by solid arrows, radial and azimuthal components of the residual velocity field - by dashed arrows. Solid curves of different thickness - the thickest, the thinnest, and intermediate - tracing the azimuthal locations of the maxima, minima and zero values of the perturbed surface density defined at every radius are denoted by $\tilde{\sigma}_{\max }, \tilde{\sigma}_{\min }$, and $0(\tilde{\sigma})$ respectively. $A$ and $C$ denote anticyclones and cyclones correspondingly. a) The angular velocities of a disc $(\Omega(r))$ and of the spiral pattern $\left(\Omega_{p h}\right)$ in the laboratory reference frame. The dashed line represents the corotation circle. b) The residual velocity field, which is the result of the subtraction of the rotation velocity from the full velocity field. Dotted lines show the boundaries of vortices. c) The velocity field in the reference frame rotating with the angular velocity $\Omega_{p h}$ of the two-armed spiral pattern. Only two anticyclones can be seen in the vicinity of the corotation circle, when the gradient of perturbed azimuthal velocity is lower than the rotation velocity gradient. d) The velocity field in the same reference frame as in the previous figure, but in the case, when the gradient of the perturbed azimuthal velocity exceeds the rotation velocity gradient. In this case one can see two cyclones and two anticyclones. Depending on the residual velocity field geometry, the domination of the cyclonic shear over the anticyclonic one can take place either near corotation, or inside (or outside) the corotation circle. Figure d) demonstrates one more possibility when four cyclones exist simultaneously - two inside and two outside the corotation circle together with two anticyclones situated at corotation.

Figure 3. Examples of trial curves used to represent the rotation curve $\left(V_{\text {rot }}(r)\right)$ in NGC 3631 are shown by solid lines together with the observed behaviour of

$a_{1}^{\text {obs }}(r)$ (triangles). The thickest line marks the rotation curve corresponding to a quasi-stationary gaseous disk. 
Figure 4. The radial dependence of $b_{3}^{\text {obs }}-b_{1}^{\text {obs }}=C_{r} \cos F_{r}$ and $b_{3}^{\text {obs }}+b_{1}^{\text {obs }}$ $=C_{\varphi} \sin F_{\varphi}$ observed in the spiral galaxy NGC 3631. An estimation of the amplitudes of the velocity components from the extrema of the presented functions gives $\max \left(C_{r}\right) \simeq \max \left(C_{\varphi}\right) \simeq 60 \mathrm{~km} / \mathrm{s}$.

Figure 5. Some parameters of the velocity field which correspond to a quasistationary regime of the vortex structure.

(a) The calculated amplitude of the radial velocity as a function of $r$.

(b) The same for the azimuthal velocity (squares) with overlaid profile of $\left|V_{\text {circ }}(r)\right|$.

Figure 6. The residual velocity field in the plane of the gaseous disc of the galaxy NGC 3631. One can see two cyclones and two anticyclones. Solid lines show some streamlines of the residual velocity field.

Figure 7. The full velocity field of the same galaxy in the reference frame rotating with the angular velocity of the two-armed spiral pattern. Overlayed squares show the position of maxima of the second Fourier harmonics of brightness map of NGC 3631 in $\mathrm{H}_{\alpha}$ line. Thin circle shows the position of the corotation. Presented streamlines (solid curves) were calculated using reverse "time" direction that allows to reveal separatrices evidently. Two cyclones and two anticyclones are enveloped by close streamlines. Other two cyclones are not enveloped by close streamlines. The anticyclone centers lie almost at corotation; the cyclone centers lie outside corotation Cyclones lie in the vicinity of spiral arms, anticyclones are situated between them. The agreement of the vortices position with theoretical predictions (scheme in Fig. 2d) is very good. 


\section{References}

[1] V. L. Afanasiev and A. M. Fridman, Astron. Lett. 19 (1993) 319.

[2] A. M. Fridman, O. V. Khoruzhii, V. V. Lyakhovich, V.S. Avedisova, O. K. Sil'chenko, A. V.Zasov, A. S. Rastorguev, V. L. Afanasiev, S. N. Dodonov and J. Boulesteix, Astrophys. Space Sci. 252 (1997) 115.

[3] G. Contopoulos, Astrophys. J. 181 (1973) 657.

[4] P. V. Baev, Yu. N. Makov and A. M. Fridman, Sov. Astron. Lett. 13 (1987) 406.

[5] G. Bertin and C. C. Lin, Spiral structure in galaxies: a density wave theory (The MIT Press; Cambridge, Massachusetts; London, England, 1995).

[6] A. M. Fridman, O. V. Khoruzhii, A. V.Zasov, O. K. Sil'chenko, A. V. Moiseev, A. N. Burlak, V.L. Afanasiev, S. N. Dodonov and J. Knapen, Astron. Lett 24 (1998) 764.

[7] V. V. Lyakhovich, A.M. Fridman, O. V. Khoruzhii, Astron. Rep. 40 (1996) 18.

[8] A. M. Fridman, O. V. Khoruzhii, V. V. Lyakhovich, O. K. Sil'chenko, A. V. Zasov, V.L.Afanasiev, S. N. Dodonov and J. Boulesteix, Astron. \& Astroph. (submitted).

[9] V. V. Lyakhovich, A. M. Fridman, O. V. Khoruzhii and A. I. Pavlov, Astron. Rep. 41 (1997) 447.

[10] B. Lindblad and R. G. Langebartel. Stockholms Observatoriums Annaler, Band 17 (1953) 6.

[11] A. Fridman, ASP Conference Series, 66 (1994) 15.

[12] D. Lynden-Bell, Barred galaxies and circumnuclear activity: proceedings of the Nobel Symposium 98, eds. A. Sandquist \& P. O. Lindblad (Springer-Verlag, Berlin, Heidelberg, New-York, 1996).

[13] G. Contopoulos, J. Hunter, and M. England, to be published.

[14] A. M. Fridman, $\quad$ O. V. Khoruzhii, E. V. Polyachenko, A. V.Zasov, O. K. Sil'chenko, A. V. Moiseev, A. N. Burlak, V. L. Afanasiev, S. N. Dodonov and J. Knapen, to be published.

[15] A. A. Sumin, A. M. Fridman, and V. A. Haud, Pis'ma Astron. Zh. 17 (1991) 698. 
a)

b)

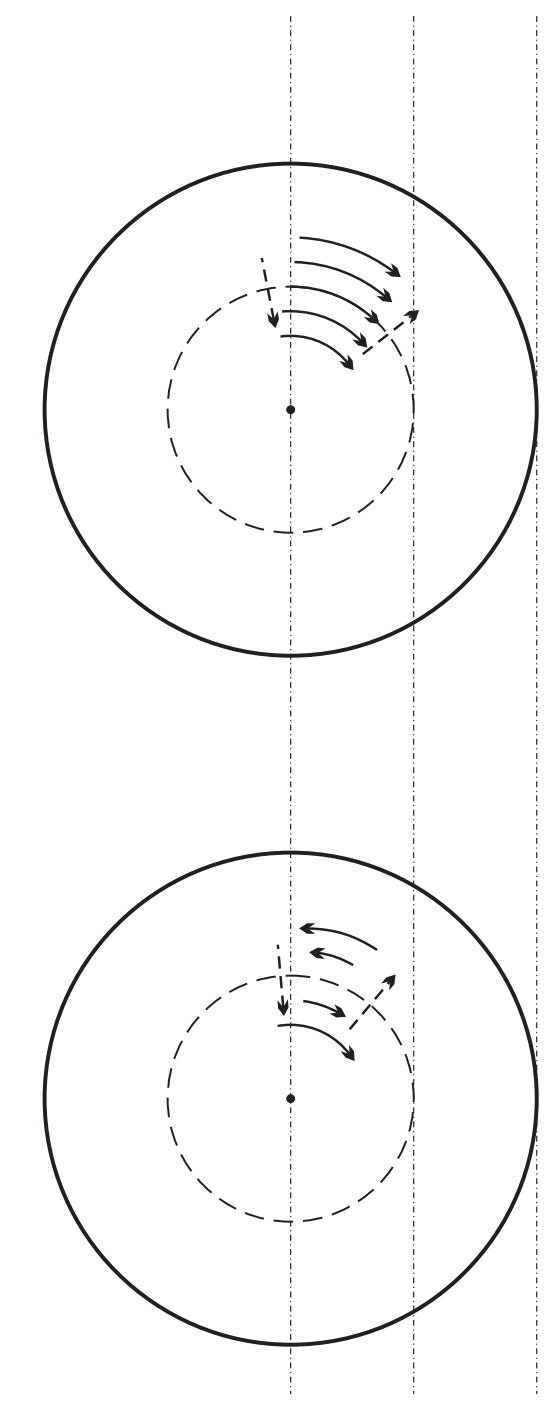


a)

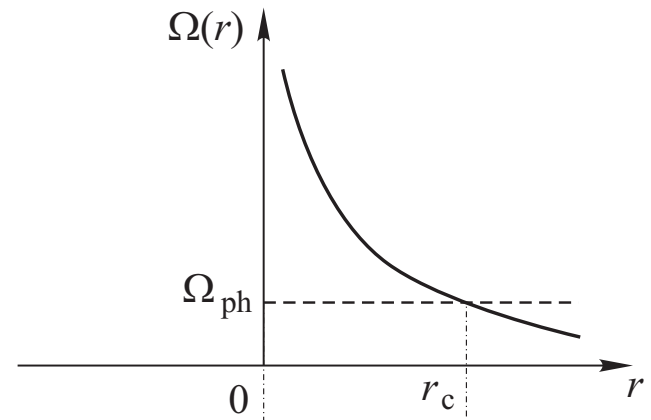

b)

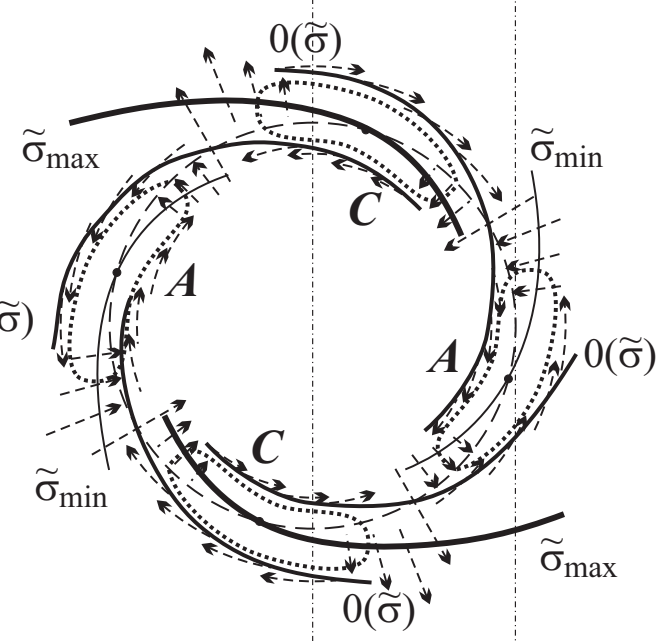

c)

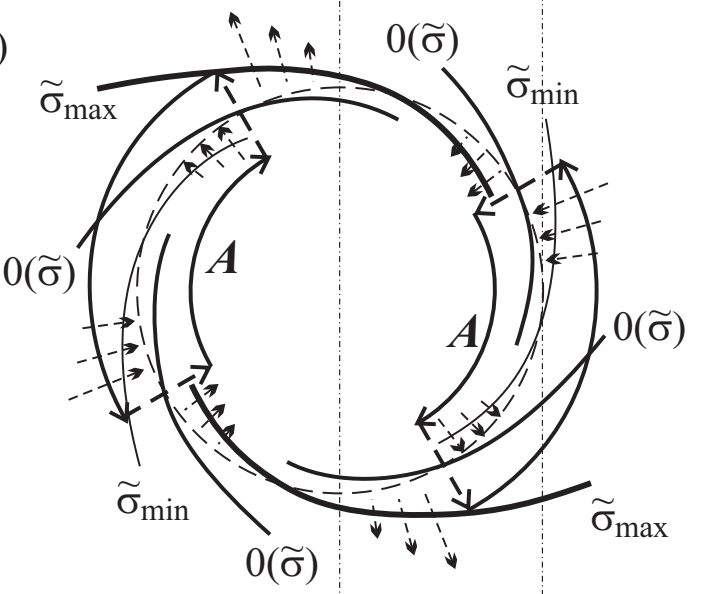

d)

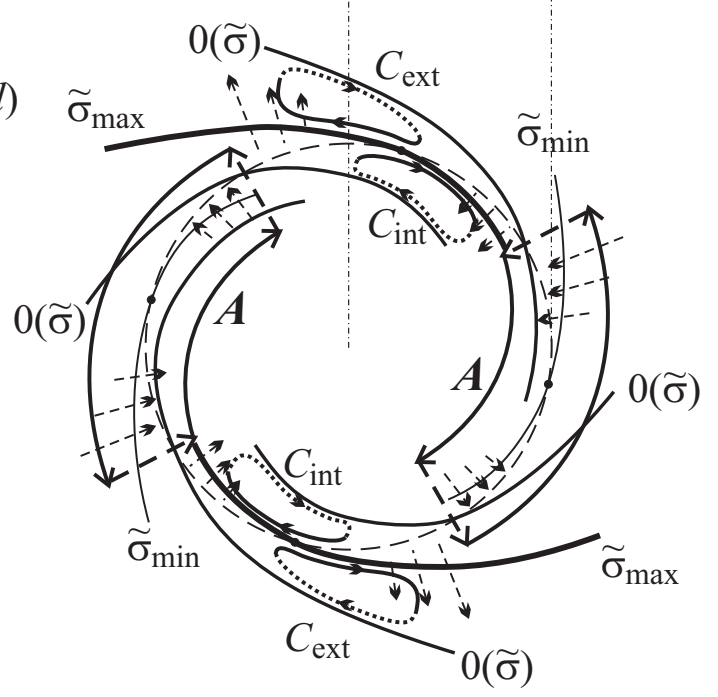




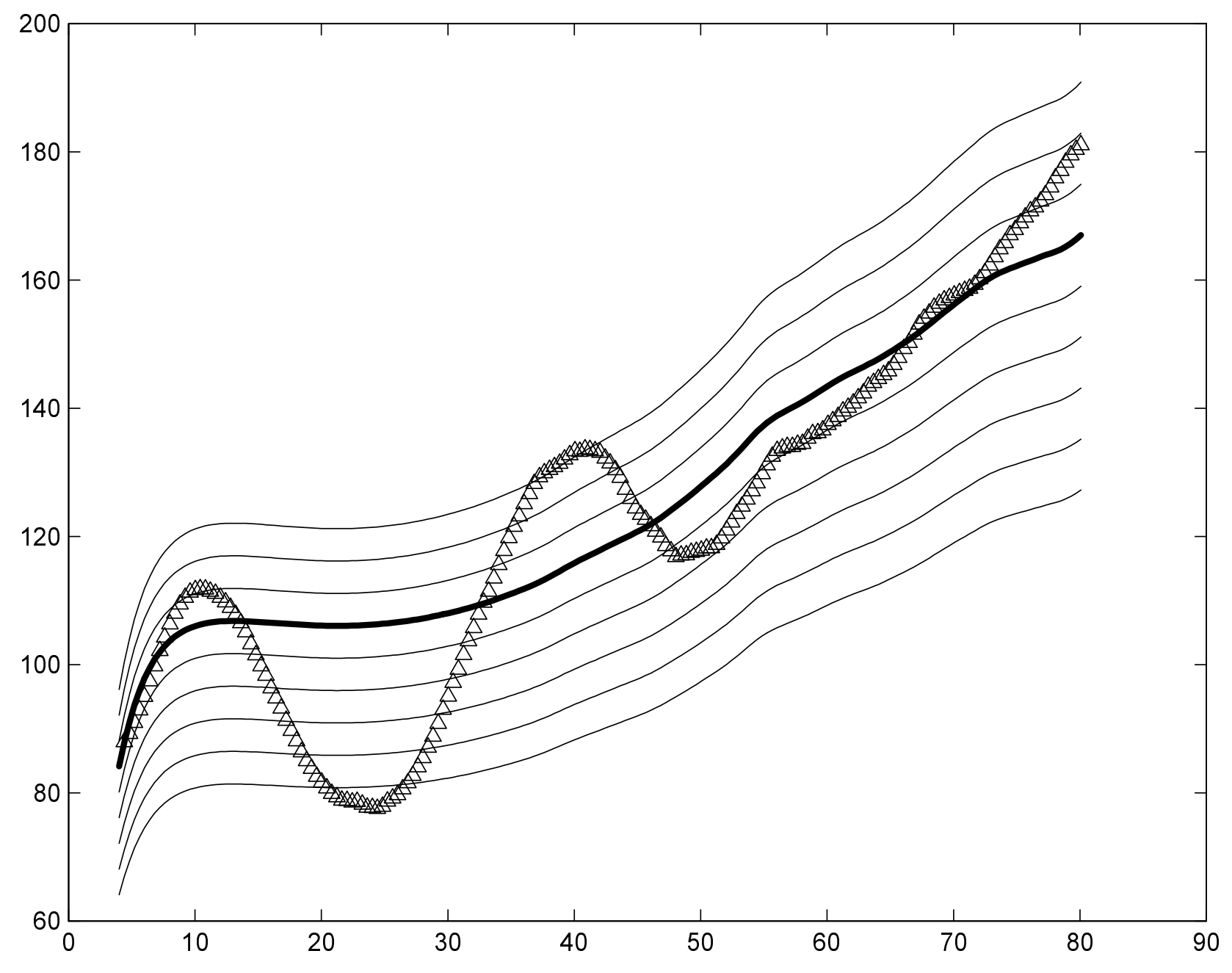



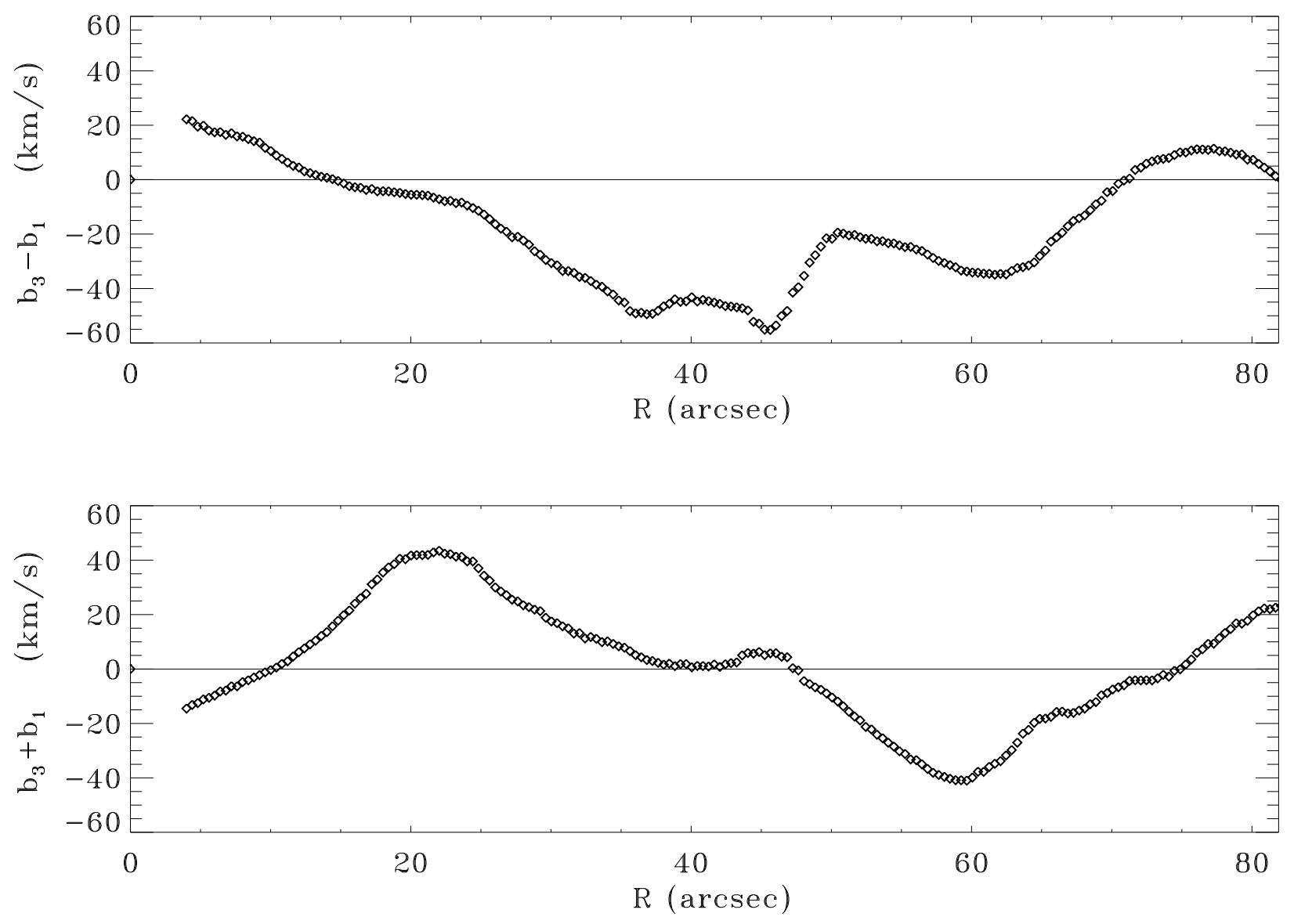
a)

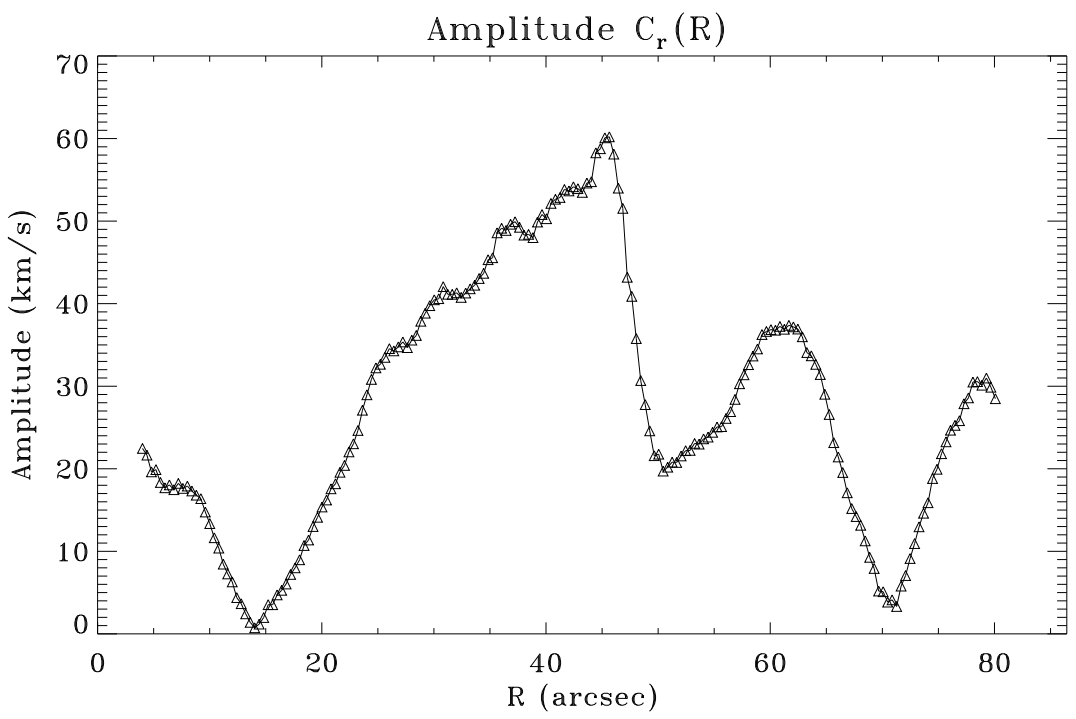

b)

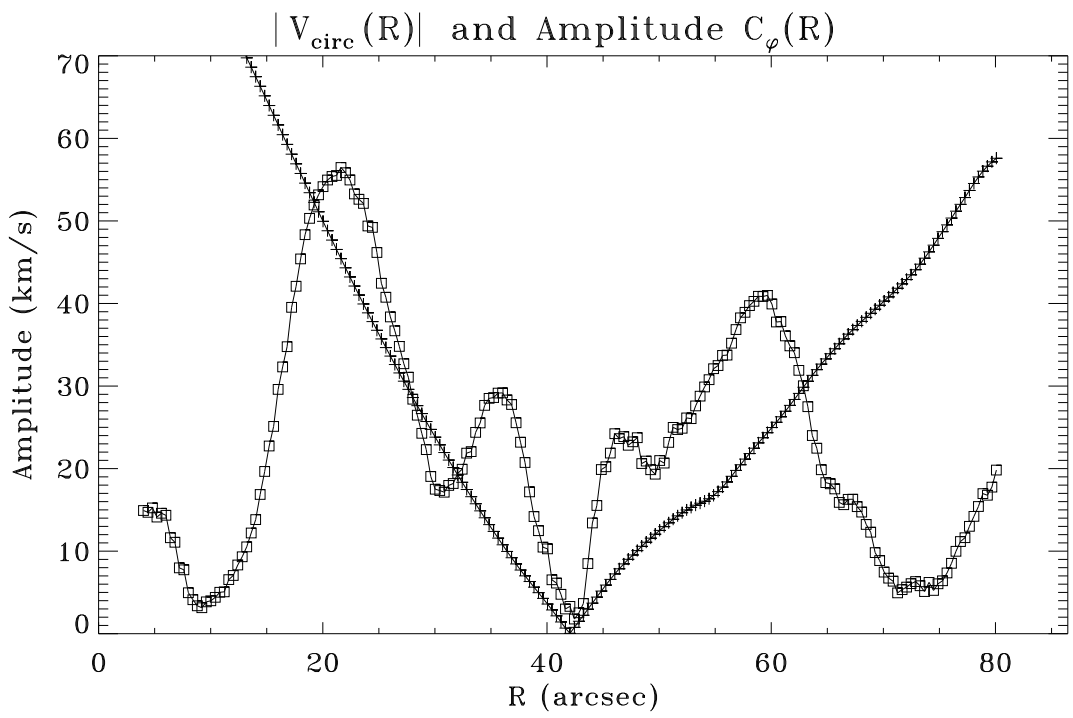




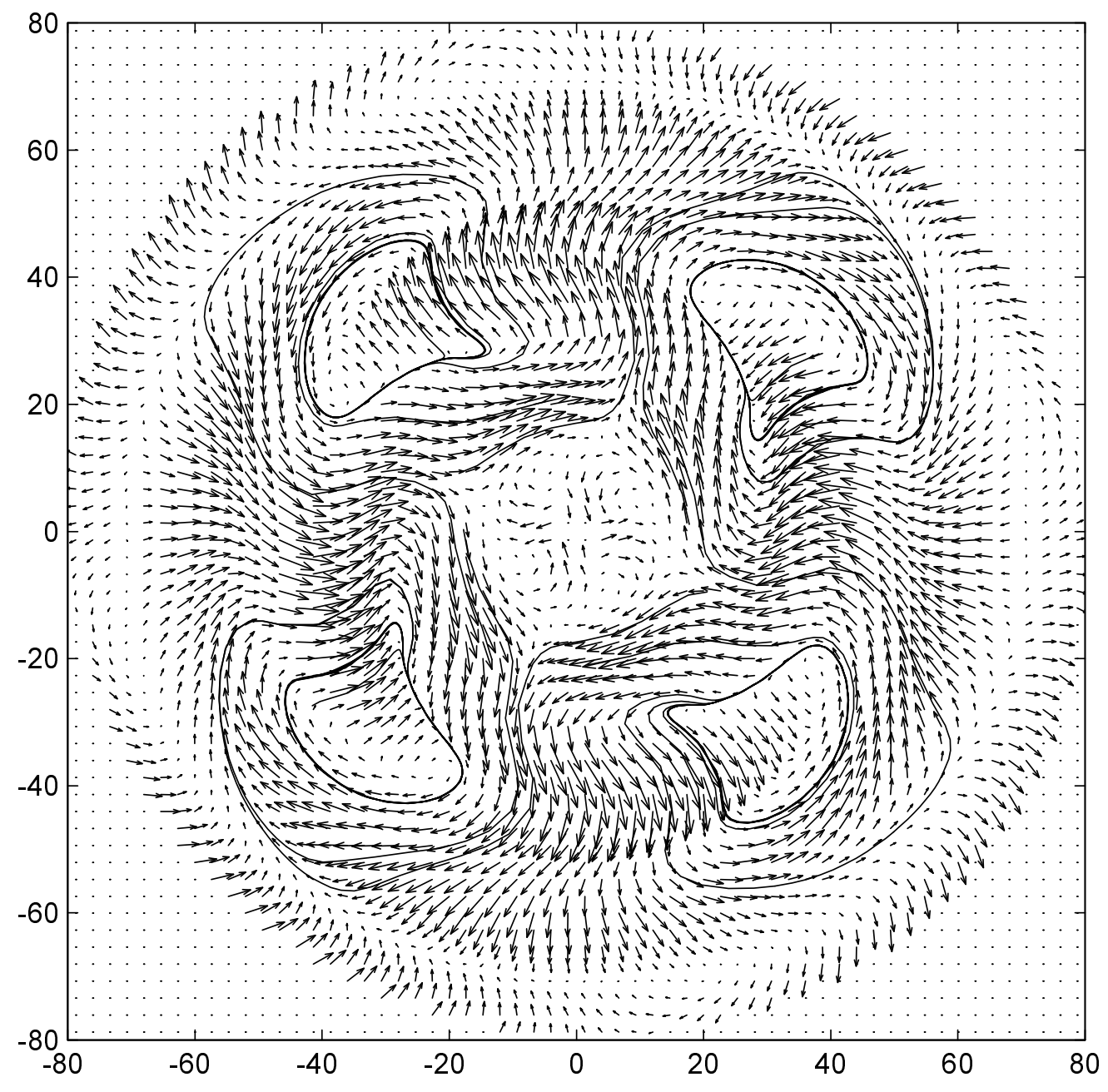




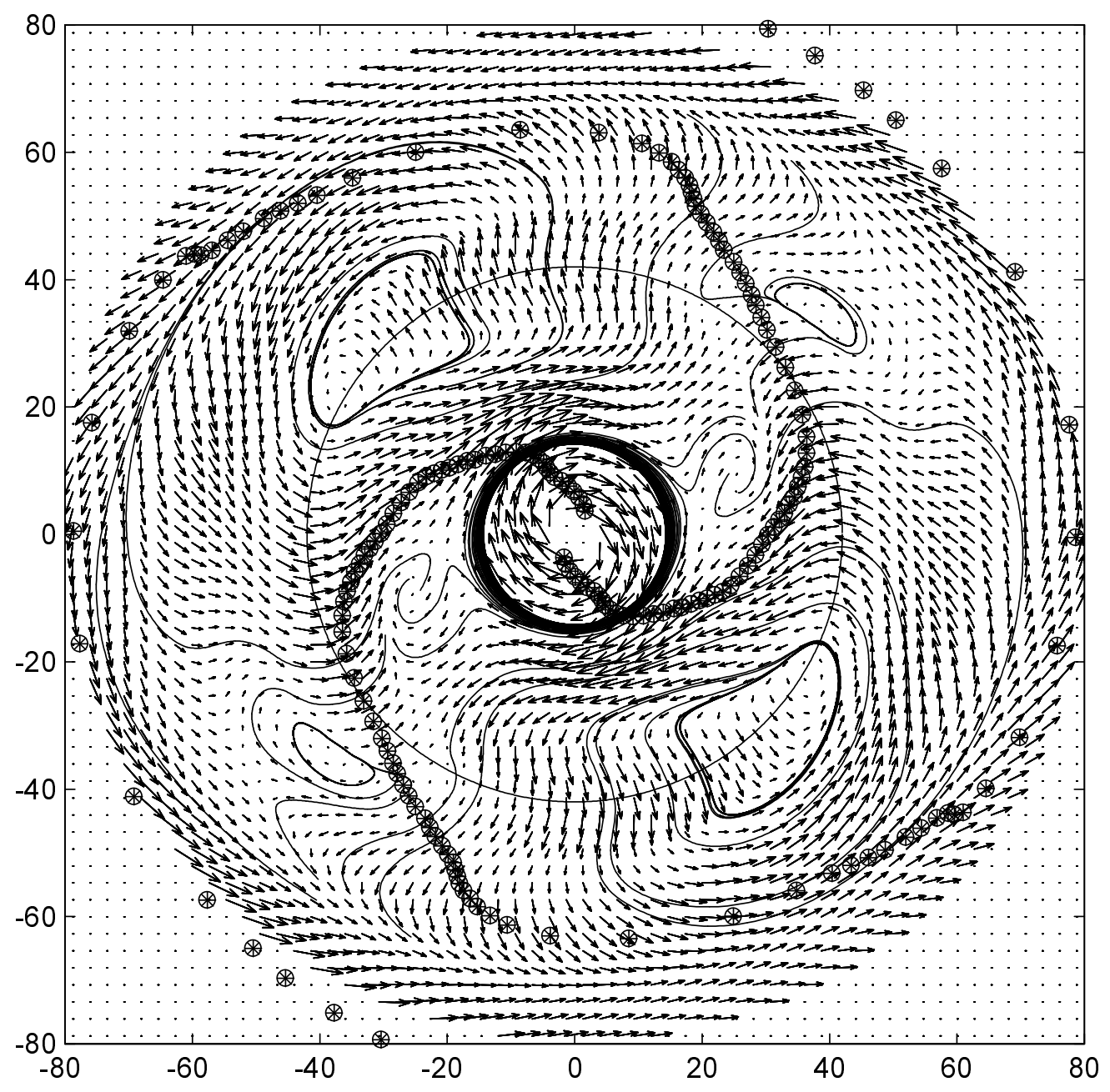

